\section{Celebrating Leadership}

A cross the country, in every province from coast to coast, dietitians demonstrate their professional leadership skills, advancing health and nutrition in varied ways. Most recently we have seen a number of forums where dietitians are actively leading in both highly visible and less visible ways. Nutrition Month, Dietitian's Day, local and regional educational days, and conferences are among these forums. We have witnessed numerous examples of dietitians advancing health and nutrition through social and traditional forms of media and by conducting informative presentations. While behind the scenes, others planned effective messaging and developed and implemented resources and educational strategies for diverse populations. Throughout my professional experience I have often, with awe, reflected upon the impressive talent, creativity, drive, and professional competence of the members of this profession.

In June we will have the opportunity once again to marvel at and celebrate dietitians as leaders when we come together in Quebec at our national conference. Three directors will be leaving the Board after 3 years of dedicated leadership service to members. Phyllis Marsch, Nathalie Savoie, and Laurie Wadsworth have each contributed their significant talents, skills, and experience to help lead the profession on its continuing path to achieving Vision 2020. We have much to thank them for.

(Can J Diet Pract Res. 2015; 76:2)

(DOI: 10.3148/cjdpr-2015-011)

Published at dcjournal.ca on 19 May 2015.
Chair's Message

MESSAGE DE LA PRÉSIDENTE

\section{Célébrer le leadership}

$\mathrm{P}$ artout au pays, dans chaque province et d'un océan à l'autre, des diététistes font preuve de leadership en promouvant de différentes façons la santé et la nutrition. Récemment, des forums ont vu le jour, et ce sont des diététistes, dans des rôles plus ou moins visibles, qui les dirigent. Le Mois de la nutrition, la Journée des diététistes, les journées de formation locales et régionales, et les congrès sont des exemples de ces forums. Par ailleurs, nous avons été témoins de nombreux exemples de diététistes faisant la promotion de la santé et de la nutrition au moyen de médias divers, sociaux ou traditionnels, et de présentations informatives. Simultanément, d'autres diététistes étaient derrière la caméra pour planifier des messages efficaces, et créer et mettre en œuvre des ressources et stratégies d'éducation destinées à diverses populations. À toutes les étapes de mon parcours professionnel, j'ai pu admirer l'incroyable talent, la créativité, le dynamisme et la compétence des membres de cette profession.

En juin, nous aurons de nouveau la chance de nous émerveiller du leadership dont font preuve les diététistes et de le célébrer à l'occasion de notre congrès national, à Québec. Trois administratrices quitteront le conseil d'administration après trois ans de dévoués services aux membres. Grâce à leurs spectaculaires talents, compétences et expériences, Phyllis Marsch, Nathalie Savoie et Laurie Wadsworth ont chacune contribué à l'essor de la profession et à la poursuite de l'objectif qu'est Vision 2020. Nous leur devons beaucoup.

(Rev can prat rech diétét. 2015; 76:2)

(DOI: 10.3148/cjdpr-2015-011)

Publié au dcjournal.ca le 19 mai 2015.

\begin{tabular}{|c|c|}
\hline 2014-2015 & Melanie Morris (Chair) \\
\hline Board of & Toronto, ON \\
\hline Directors & $416-447-1771$ \\
\hline Cons & Laurie A. Wadsworth \\
\hline d'administration & $902-867-2190$ \\
\hline
\end{tabular}

Patti Simpson Yarmouth, NS 902-742-6528

Doug Cook
Toronto, ON
416-413-9095
Toronto, ON
416-413-9095
Phyllis Marsch Winnipeg, $\mathrm{MB}$ 204-275-1918

Sarah Hewko Edmonton, $\mathrm{AB}$ 780-628-2080

Michelle McLearn
Middleton, NS
902-825-6160 Ext. 364
Nicole Spencer
Vancouver, BC
$604-564-6534$

Nathalie Savoie Montreal, QC 514-284-1092

Chief Executive Officer Marsha Sharp 416-642-9314 